\title{
Einbezug von Alter und Sprachkontaktdauer in die Wortschatzdiagnostik bei Mehrsprachigkeit
}

\author{
Birgit Ehl ${ }^{1}$ id und Michael Grosche ${ }^{1}$ (D) \\ 'Institut für Bildungsforschung, School of Education, Bergische Universität Wuppertal
}

\begin{abstract}
Zusammenfassung: Für die expressive Wortschatzdiagnostik mehrsprachiger Kinder im Grundschulalter fehlen Auswertungsansätze, die die heterogenen Bedingungen des Mehrspracherwerbs berücksichtigen. Solche Auswertungsansätze werden im Bildungs- und Gesundheitswesen gebraucht, um eine bessere Orientierung zu erhalten, welche mehrsprachigen Kinder sprachlich besonders auffällig sind. Die vorliegende Studie prüft, ob das Alter der Kinder und deren Kontaktdauer zur Umgebungssprache als Bestandteile mehrspracherwerbssensibler Normen für Wortschatzleistungen in der Umgebungssprache in Betracht kommen. Bei einer Stichprobe von 451 mehrsprachigen Grundschulkindern hatten das Alter und die Kontaktdauer einen bedeutsamen Einfluss auf den Wortschatz in der Umgebungssprache. Darüber hinaus zeigten sich differentielle Zusammenhänge. Kinder mit kürzerer Kontaktdauer unterschieden sich im Wortschatz stärker von Kindern, die z. B. ein Jahr älter und ein Kontaktjahr weiter fortgeschritten waren, als Kindern mit längerer Kontaktdauer. Insgesamt weisen die Ergebnisse darauf hin, dass die Entwicklung von mehrspracherwerbssensiblen Normen die Identifikation von sprachlich besonders auffälligen Kindern verbessern könnte.
\end{abstract}

Schlüsselwörter: Wortschatz, Mehrsprachigkeit, Grundschule, Diagnostik, Prädiktion.

Considering Age and Length of Exposure to the Environmental Language in Vocabulary Assessment in Multilingualism

Abstract: There is a dearth of evaluation approaches for the assessment of expressive vocabulary of multilingual school-aged children that take the heterogeneous conditions of multilingual language acquisition into account. Such evaluation approaches are needed in education and healthcare to provide better orientation which multilingual children have noticeable language problems. This study investigates whether age and length of exposure (LoE) to the environmental language can be considered as components of norms for vocabulary assessments. In a sample of 451 multilingual primary students, age and LoE were significant predictors of environmental vocabulary. Furthermore, differential relationships were found. Children with a shorter LoE differed more in vocabulary from children who were, for example, one year older and one contact year more advanced than children with a longer LoE. Overall, the results indicate that the development of norms of multilingual children that take age and LOE into account could improve the identification of multilingual children with noticeable language problems.

Keywords: vocabulary, bilingualism, elementary school, diagnosis, prediction.

Für den Bildungserfolg und die sozio-emotionale Entwicklung sind umfassende Sprachkompetenzen von zentraler Bedeutung (Lowe, Henry, Müller \& Joffe, 2018; Reiss, Weis, Klieme \& Köller, 2019). Daher gehört die Beobachtung der Sprachentwicklung, die Feststellung des Sprachentwicklungsstandes sowie die Identifikation von Sprachauffälligkeiten und die Auswahl und Durchführung von Förder- und Therapiemaßnahmen zu den interdisziplinären Aufgaben von Akteurinnen und Akteuren im Bildungs- und Gesundheitswesen (AWMF, 2011; KMK, 2019; Schneider et al., 2012). Um im komplexen Bereich Sprache den Entwicklungsstand angemessen feststellen und Auffälligkeiten identifizieren zu können, wird neben der Interdisziplinarität zudem ein multimethodales Vorgehen gefordert: Es sollen sowohl informelle Verfahren wie Befragungen und Beobachtungen als auch formelle Verfahren wie normierte und standardisierte Tests zur Prüfung sprachlicher Leistungen in allen sprachlichen Bereichen sowie der Sprachverarbeitung zum Einsatz kommen (AWMF, 2011; KMK, 2019; Melzer, Rißling, Lehmkuhl \& Petermann, 2018). Informelle Verfahren liefern wichtige Informationen von Eltern und Lehrkräften zur Sprachbiografie sowie zum Sprachverhalten in natürlichen Kontexten. Standardisierte, bezugsgruppenorientierte Verfahren ermöglichen einen sozialen Vergleich der Leistung mit einer Bezugsgruppe, die vergleichbare Er- 
werbsbedingungen hat, wie beispielsweise eine Altersgruppe. Sie gelten deshalb als „basales Bezugssystem“ (Kany \& Schöler, 2009, S. 58).

Bei mehrsprachigen Kindern ist die Sprachdiagnostik nochmals komplexer. Als mehrsprachig werden in diesem Beitrag Kinder bezeichnet, die mit mehr als einer Sprache aufwachsen. Damit ist gemeint, dass sie zwei oder mehr Sprachen (Umgebungs- und Nichtumgebungssprachen) in einem natürlichen Kontext erwerben, unabhängig davon, ob der Erwerb mit der Geburt oder zu einem späteren Zeitpunkt vor dem Schuleintritt beginnt (Bialystok, 2001; Hammer et al., 2014). In die Sprachdiagnostik mehrsprachiger Kinder sollen bestenfalls Informationen zum Erwerb sowie zu den Kompetenzen in allen Sprachen einfließen (Grosjean, 2016; Lüke, Starke \& Ritterfeld, 2020; Marinis, Armon-Lotem \& Pontikas, 2017), weil die Sprachkompetenzen zum einen von den Spracherwerbsbedingungen abhängig sind und zum anderen miteinander in Beziehung stehen. Beispielsweise für die Erfassung von Wortschatzleistungen sind daher zur Berücksichtigung beider Sprachen Wortschatztests mit parallelen Sprachversionen besonders hilfreich, um den konzeptuellen Wortschatzumfang ermitteln zu können. Hierbei werden Items korrekt gewertet, wenn sie in mindestens einer der beiden Sprachen benannt werden. Im konzeptuellen Wortschatz können mehrsprachige Kinder mit einsprachigen Kindern vergleichbare Leistungen zeigen (Ehl, Bruns \& Grosche, 2020). Insbesondere für die diagnostische Fragestellung, ob eine Sprachentwicklungsstörung besteht, ist der Einbezug aller Sprachen hilfreich, da eine Sprachentwicklungsstörung u. a. daran zu erkennen ist, dass sie sich in allen Sprachen des Kindes manifestiert (Håkansson, Salameh \& Nettelbladt, 2003).

Allerdings sehen sich die Untersucherinnen und Untersucher für die Diagnostik in den Nichtumgebungssprachen mit erheblichen Herausforderungen konfrontiert. Für viele Sprachen und sprachliche Bereiche fehlen Diagnostikverfahren. Zudem sind häufig auf Seiten der Untersuchungsleitung Kenntnisse in den betreffenden Nichtumgebungssprachen erforderlich (Teoh, Brebner \& McAllister, 2018).

Als Ausweg aus diesem Dilemma werden zur Zeit zahlreiche Forschungsbemühungen in Nordamerika und Europa unternommen. Sie zielen zum einen auf die Berücksichtigung beider Sprachen ab und zum anderen auf Alternativen, um die Umgebungssprache unter Berücksichtigung der mehrsprachigen Erwerbsbedingungen zu untersuchen. Zu diesen Alternativen zählen beispielsweise separate Normen für mehrsprachige Kinder (Paradis, 2016). Diese separaten Normen sollen an einer Normstichprobe mehrsprachiger Kinder mit vergleichbaren Erwerbsbedingungen ermittelt werden (im Folgenden mehrspracherwerbssensible Normen) (Gathercole, Tho- mas, Roberts, Hughes \& Hughes, 2013; Peña, Bedore \& Fiestas, 2013). Allerdings können manche familiäre Bedingungen die Sprachleistungen beeinflussen, die nur schwer in Normen realisiert werden können, z. B. das Bildungsniveau der Eltern (Vernon-Feagans, Bratsch-Hines, Reynolds \& Willoughby, 2020) und die Qualität des Sprachinputs (Daskalaki, Blom, Chondrogianni \& Paradis, 2020; Unsworth, 2016b). Im Gegensatz dazu in Normen realisierbar und diagnostisch sinnvoll sind das chronologische Alter des Kindes und dessen Kontaktdauer zur Umgebungssprache (im Folgenden nur Kontaktdauer), d. h. die Differenz aus dem Alter zum Testzeitpunkt und dem Alter zu Erwerbsbeginn (Schulz \& Tracy, 2011). In der Kontaktdauer unterscheiden sich mehrsprachige Kinder untereinander (Paradis, 2018; Thordardottir, 2011). Manche beginnen mit dem Erwerb mehrerer Sprachen mit der Geburt, und andere haben erst ab dem Eintritt in eine Kindertagesstätte regelmäßige Erwerbsgelegenheiten zu einer zweiten Sprache, um nur zwei von zahlreichen Varianten von Erwerbskonstellationen zu nennen. Die Kontaktdauer und das Alter werden daher als Bestandteile mehrspracherwerbssensibler Normen vorgeschlagen (Neugebauer \& Becker-Mrotzek, 2015; Paradis, 2010).

Mehrspracherwerbssensible Normen sind hilfreich, da sie eine wesentliche Voraussetzung der bezugsgruppenorientierten Norm, also die möglichst vergleichbaren Erwerbsbedingungen, erfüllen. Erste empirische Befunde zu mehrspracherwerbssensiblen Normen weisen auf ihr Potential hin, da ein auffälliger Befund nur noch in einem geringeren Ausmaß auf unvergleichbare Erwerbsbedingungen zurückgeführt werden kann (Paradis, Schneider \& Sorensen Duncan, 2013). Sie könnten eine bessere Orientierung liefern, welche mehrsprachigen Kinder in ihrer Sprachentwicklung durch Akteurinnen und Akteure im Bildungs- und Gesundheitswesen besonders zu beobachten und ggf. für eine tiefergehende Abklärung der sprachlichen Auffälligkeiten vorzuschlagen sind. Zudem könnten sie helfen, realistischere Erwartungen an die sprachlichen Leistungen mehrsprachiger Kinder in ihrer Umgebungssprache zu stellen und der defizitorientierten Sichtweise, die aus dem Vergleich mit einsprachigen Kindern resultiert, entgegenzuwirken. Daher werden sie als "ideal“ (Settinieri \& Jeuk, 2019, S. 10) bzw. als „wichtiger Schritt in Richtung theoretisch und empirisch fundiertem sowie standardisiertem Umgang mit Mehrsprachigkeit" (Reitenbach, Schastak \& Rauch, 2018, S. 141) bezeichnet.

Bisher existieren in Deutschland nur vereinzelte sprachdiagnostische Verfahren für verschiedene sprachliche Bereiche, die eine Norm zum Vergleich mit mehrsprachigen Kindern vorhalten (z. B. Lenhard \& Lenhard, 2017; Schulz \& Tracy, 2011; Seifert, Paleczek, Schwab \& Gasteiger-Klicpera, 2017). Für den expressiven Wort- 
schatz in Deutsch existiert bisher kein Verfahren mit mehrspracherwerbssensiblen Normen (Geist, 2017). Dabei ist ein differenzierter Wortschatz insbesondere für Schulkinder wichtig, da er die Grundlage der sprachlichen und schriftsprachlichen Kompetenzen darstellt (McElvany, El-Khechen, Schwabe \& Kessels, 2016; Torppa et al., 2007). Auf diese Forschungslücke geht der vorliegende Beitrag ein. Wir prüfen, inwiefern sich die Erwerbsbedingungen Alter und Kontaktdauer auch für expressive Wortschatzleistungen in der Umgebungssprache mehrsprachiger Kinder als Bestandteile von mehrspracherwerbssensiblen Normen eignen. Dazu berichten wir zunächst über empirische Befunde zum Einfluss des Alters und der Inputmenge, zu der auch die Kontaktdauer zählt, auf die Wortschatzleistungen.

\section{Empirische Befunde zum Einfluss des Alters auf den Wortschatz}

Aufgrund der kognitiven Prozesse, die beim Spracherwerb ablaufen, ist anzunehmen, dass ältere mehrsprachige Kinder mit reiferen kognitiven Ressourcen höhere Wortschatzleistungen aufweisen als jüngere mehrsprachige Kinder (Wong Fillmore, 2000). Entsprechende Befunde sind jedoch inkonsistent. Geist (2017) zeigte für 5bis 6-jährige mehrsprachige Kinder mit einem Erwerbsbeginn zur Umgebungssprache ab zwei Jahren keinen signifikanten Zusammenhang zwischen dem Alter und den expressiven Wortschatzleistungen in der Umgebungssprache. Auch bei 4- bis 11-jährigen mehrsprachigen Kindern mit einem Erwerbsbeginn vor und ab 4 Jahren erwies sich das Alter nicht als signifikante Kovariate für die rezeptiven Wortschatzleistungen in der Umgebungssprache (Unsworth, 2016a). Demgegenüber stehen Befunde von mehrsprachigen Kindern unterschiedlichen Alters, die einen signifikanten Einfluss des Alters auf rezeptive Wortschatzleistungen in unterschiedlichen Umgebungssprachen zeigten (Gathercole \& Thomas, 2009 für Kinder mit gleichzeitigem oder aufeinanderfolgendem Erwerb der Sprachen; Paradis, 2011 für mehrheitlich Kinder mit einem Erwerbsbegin ab ca. 2 bis 3 Jahren; Thordardottir, 2019 für mehrspachige Kinder mit einem Erwerbsbeginn vor und ab 3 Jahren). Allerdings wurde lediglich in drei Studien (Paradis, 2011; Thordardottir, 2019; Unsworth, 2016a) differenziert der Einfluss der Kontaktdauer bei der Bestimmung des Einflusses des Alters kontrolliert. Da beide Faktoren stark miteinander konfundiert sind, könnten in den anderen Studien die berichteten Einflüsse auch auf die Kontaktdauer zurückführbar sein.

\section{Empirische Befunde zum Einfluss der Inputmenge auf den Wortschatz}

Für den Erwerb einer Sprache ist es wichtig, dass ein Kind häufig in Kontakt mit Sprechern der Zielsprache ist (Wong Fillmore, 2000). Die Inputmenge wird in empirischen Studien zum Einfluss auf den Wortschatzumfang auf sehr unterschiedliche Arten operationalisiert, z. B. als Angabe, die das Verhältnis der Sprachen im Input des Kindes angibt (Gathercole \& Thomas, 2009; Thordardottir, 2011; Unsworth, 2016a), oder als Kontaktdauer in Monaten (Geist, 2017; Paradis, 2011). In allen Studien zeigten sich entweder ein signifikanter positiver Zusammenhang zwischen der Kontaktdauer und dem expressiven Wortschatz (Geist, 2017) oder ein signifikanter Einfluss der Inputmenge auf den rezeptiven Wortschatz in unterschiedlichen Umgebungssprachen und für mehrsprachige Kinder unterschiedlichen Alters sowie sowohl gleichzeitigem als auch aufeinanderfolgendem Erwerbsbeginn zur Umgebungssprache (Gathercole \& Thomas, 2009; Paradis, 2011; Thordardottir, 2011; Unsworth, 2016a).

Zwei Studien verglichen die Stärke des Einflusses des chronologischen Alters und der Inputmenge (Thordardottir, 2019) bzw. der Kontaktdauer (Paradis, 2011) auf die rezeptiven Wortschatzleistungen in verschiedenen Umgebungssprachen. Beide Studien stimmen darin überein, dass der Einfluss der Inputmenge bzw. der Kontaktdauer größer ist als der Einfluss des Alters.

Darüber hinaus untersuchten Cobo-Lewis, Pearson, Eilers und Umbel (2010) differentielle Zusammenhängen zwischen dem Wortschatz und dem Alter (operationalisiert als Bildungsetappe), in Abhängigkeit von der Inputmenge (operationalisiert als familiärer Sprachgebrauch). Die rezeptiven und expressiven Wortschatzleistungen von Kindern, die zu Hause nur die Nichtumgebungssprache sprachen, waren geringer als von Kindern, die zu Hause sowohl die Umgebungs- als auch die Nichtumgebungssprache sprachen, entwickelten sich dafür aber signifikant schneller.

Die beschriebenen empirischen Befunde unterstreichen einheitlich die Schlüsselrolle der Inputmenge für die Wortschatzleistungen (Hammer et al., 2014).

\section{Fragestellungen und Hypothesen}

Damit Akteurinnen und Akteure im Bildungs- und Gesundheitswesen eine bessere Orientierung erhalten, welche mehrsprachigen Kinder sprachlich besonders auffällig sind und daher in ihrer sprachlichen Entwicklung besonders beobachtet bzw. für eine tiefergehende Diagnostik empfohlen werden sollen, wären mehrspracherwerbssensible Normen hilfreich. Jedoch ist bisher nicht geklärt, 
inwiefern das Alter und die Kontaktdauer als Bestandteile mehrspracherwerbssensibler Normen für expressive Wortschatzleistungen mehrsprachiger Grundschulkinder in Betracht kommen. Damit gerechtfertigt ist, dass Altersnormen für mehrsprachige Kinder zusätzlich die Kontaktdauer berücksichtigen, müsste nach der Berücksichtigung des Alters noch ein zusätzlicher Varianzanteil in den Wortschatzleistungen für die Kontaktdauer nachgewiesen werden. Differentielle Zusammenhänge zwischen dem Alter und den expressiven Wortschatzleistungen abhängig von der Kontaktdauer würden mehrspracherwerbssensible Normen noch stärker rechtfertigen.

Daher untersuchen wir erstens, ob die Kontaktdauer und das Alter einen Effekt auf die expressiven Wortschatzleistungen mehrsprachiger Grundschulkinder haben, wie stark die Effekte im Vergleich sind und ob die Hinzunahme der Kontaktdauer als zusätzliche Erwerbsbedingung zum Alter die Vorhersage der expressiven Wortschatzleistungen verändert. Wir nehmen an, dass sowohl das Alter als auch die Kontaktdauer einen positiven Effekt auf die expressiven Wortschatzleistungen haben, weil ältere Kinder durch ihre höhere kognitive Reife und eine längere Kontaktdauer in der Regel durch eine größere Inputmenge zu höheren Wortschatzleistungen führen. Zudem prognostizieren wir, dass die Hinzunahme der Kontaktdauer zusätzlich zum Alter die Vorhersage der expressiven Wortschatzleistungen erhöht.

Zweitens analysieren wir, ob der Effekt des Alters auf die expressiven Wortschatzleistungen unter dem Einfluss der Kontaktdauer variiert. Hierbei prognostizieren wir, dass die Kontaktdauer den Effekt des Alters moderiert, indem sich mehrsprachige Kinder mit einer kürzeren Kontaktdauer stärker von Kindern unterscheiden, die z. B. ein Jahr älter und ein Kontaktjahr weiter fortgeschritten sind, als Kinder mit einer längeren Kontaktdauer (CoboLewis et al., 2010). Würden diese Hypothesen bestätigt werden, würden wir mit unserer Studie gute empirische Argumente zur Entwicklung mehrspracherwerbssensibler Normen für expressive Wortschatzleistungen mehrsprachiger Grundschulkinder in ihrer Umgebungssprache liefern.

\section{Methode}

\section{Untersuchungsinstrumente}

Für die Erhebung der expressiven Wortschatzleistungen in der Umgebungssprache Deutsch wurden wie auch in anderen Studien (Ertanir, Kratzmann \& Sachse, 2019; Hamann \& Abed Ibrahim, 2017) zwei häufig verwendete, standardisierte Verfahren eingesetzt, die für einsprachige
Kinder konzipiert sind, da keine entsprechenden Verfahren für mehrsprachige Kinder existieren. Dabei handelt es sich um den altersadäquaten Wortschatz- und Wortfindungstest für 6- bis 10-Jährige (WWT 6-10, Glück, 2011) und den für jüngere Kinder konzipierten Aktiven Wortschatztest für 3 bis 5-Jährige - Revision (AWST-R, KieseHimmel, 2005). Der AWST-R kam zum Einsatz, da bei den mehrsprachigen Kindern aufgrund ihrer in der Regel geringeren Inputmenge zu Deutsch Bodeneffekte in einem altersangemessenen Wortschatztest wie dem WWT 6-10 zu erwarten waren.

Der WWT 6-10 ist ein Bildbenennungstest. Die Langform in der PC-Version enthält 95 Items aus fotografischen Darstellungen von 26 Substantiven und jeweils 23 Verben, Adjektiven und Oberbegriffen. Die Normierungsstichprobe besteht aus einsprachig deutschen Kindern. Anders als im Testtitel suggeriert, beginnen die Normen bereits ab dem Alter von 5;6 Jahren und sind mit einer Ausnahme (5;6 bis 6;5 Jahre) in Halbjahresspannen aufgegliedert. Die interne Konsistenz des eindimensionalen WWT 6-10 beträgt $\alpha=.91$.

Der AWST-R ist ebenfalls ein Bildbenennungstest mit 75 Items aus fotografischen Darstellungen von 51 Substantiven und 24 Verben. Die Normierungsstichprobe besteht ebenfalls aus einsprachig deutschen Kindern. Die Altersnormen decken den Zeitraum von 3;0 bis 5;5 Jahren ab und sind in Halbjahresspannen aufgegliedert. Die interne Konsistenz des ebenfalls eindimensionalen AWST-R beträgt $\alpha=.88$.

Darüber hinaus wurde der nonverbale IQ mit der Grundintelligenztest Skala 1 - Revision (CFT 1-R, Weiß \& Osterland, 2012) erhoben und in der Langfassung als Gruppentest durchgeführt. Die interne Konsistenz des CFT 1-R beträgt $\alpha=.97$.

Des Weiteren wurden mit Hilfe eines Elternfragebogens Fragen zu personenspezifischen Informationen (Geburtsdatum, Kita-Eintritt und Schuleintritt), zum Sprachgebrauch in der Familie und zum Zeitpunkt des Beginns des regelmäßigen Kontakts zur Umgebungssprache Deutsch gestellt.

\section{Stichprobe}

Die Stichprobe bestand aus 451 mehrsprachigen Schülerinnen und Schülern (214 Mädchen, 237 Jungen) im Alter von $5 ; 10$ bis $10 ; 11$ Jahre $(M=8.01, S D=1.26)$ aus 80 Klassen in elf Grundschulen in Wuppertal. Insgesamt waren 31 nicht-deutsche Sprachen vertreten, darunter am häufigsten Türkisch (36,7\%), Russisch (9,8\%), Arabisch (7,0\%), Griechisch (6,8\%), Polnisch (6,5\%) und Kurdisch $(6,5 \%)$ (andere Sprachen jeweils $<5 \%)$. Die Kontaktdauer zu Deutsch (Anzahl der Monate seit Beginn des re- 
gelmäßigen Kontakts zu Deutsch bis zum Erhebungszeitpunkt) variierte zwischen 13 und 131 Monaten $(M=73.63$, $S D=25.38) .60 .1 \%(n=271)$ der Kinder kamen vor dem Alter von 3 Jahren regelmäßig mit der deutschen Sprache in Kontakt, für $39.9 \%(n=180)$ der Kinder begann der regelmäßige Kontakt mit dem Deutschen ab dem Alter von 3 Jahren. Die T-Werte im non-verbalen IQ lagen im Mittel kaum unter $50(M=48.86, S D=8.75 ; t(450)=-$ $2,75, p=.007, d=-0.13)$.

Um zu illustrieren, wie ein Vergleich zur Normstichprobe aus einsprachig deutschen Kindern ausfällt, wiesen wir den Rohwerten im WWT 6-10 anhand des chronologischen Alters die T-Werte laut Testmanual zu. Bei 42 Kindern war das Abbruchkriterium im WWT 6-10 erfüllt, weshalb lediglich Angaben zu 409 Kindern einfließen. Die T-Werte betragen im Mittel $30.33(S D=12.64$, Min. $=0, \operatorname{Max} .=68)$. Eine überwiegende Mehrheit von insgesamt $78.7 \%(n=322)$ hat einen T-Wert unter 40 und somit in diesem rein altersbezogenen sozialen Vergleich einen auffälligen Wortschatz. Die bereits erwarteten Bodeneffekte der mehrsprachigen Kinder im WWT 6-10 sind deutlich erkennbar. $66.0 \%(n=270)$ haben im altersbasierten Vergleich mit einsprachigen Kindern sehr geringe $\mathrm{T}$-Werte zwischen $\mathrm{O}$ und 34, auch wenn ihre Wortschatzleistungen für ihre Kontaktdauer als erwartbar zu beurteilen sein könnten. Im AWST-R können die Rohwerte lediglich mit den T-Werten für jüngere einsprachige Kinder im Alter von 5;0 bis 5;5 Jahren verglichen werden. Die T-Werte betragen im Mittel $45.12(S D=13.62$, Min. $=$ 24, Max. $=80$ ) und zeigen keine Deckeneffekte.

Als Ausschlusskriterien galten ein nonverbaler IQ mit einem T-Wert $<30$, ein diagnostizierter sonderpädagogischer Förderbedarf im Bereich Sprache, ein Beginn des Erwerb des Deutschen erst ab der Einschulung bzw. zum Erhebungszeitpunkt eine Kontaktdauer zu Deutsch von 12 Monaten oder weniger.

\section{Vorgehensweise}

Die querschnittliche Erhebung der expressiven Wortschatzleistungen fand als Einzeltest in einem ruhigen Raum in den jeweiligen Schulen statt. In einer Sitzung von 45 Minuten wurden zuerst der AWST-R und nachfolgend der WWT 6-10 von geschulten Testleiterinnen und Testleitern durchgeführt. Die türkischsprachigen Kinder wurden zusätzlich mit türkischen Sprachversionen der Wortschatztests untersucht, die Ergebnisse sind jedoch für die vorliegende Studie nicht relevant (siehe hierzu Ehl, Bruns \& Grosche, 2020). An einem separaten Tag wurde der CFT 1-R in Kleingruppen von 10 bis 15 Kindern durchgeführt.

\section{Analysen}

Die expressiven Wortschatzleistungen in Deutsch wurden operationalisiert als Summenscore der Rohwerte aus AWST-R und WWT 6-10 abzüglich drei Items, die in beiden Tests vorkamen und nur einmal in den Summenscore eingingen. Somit betrug der maximale Summenscore 167 Rohwertpunkte. Die interne Konsistenz des Summenscores beträgt $\alpha=.97$. Die Addition der Rohwerte aus beiden Wortschatztests zu einem Summenscore war bereits bei der Studienplanung vorgesehen, da für mehrsprachige Kinder deutliche Bodeneffekte im WWT 6-10 erwartet worden waren und auch nachgewiesen werden konnten. Der Summenscore aus AWST-R und WWT 6-10 ermöglicht, die Leistungen der mehrsprachigen Kinder ohne Bodeneffekte und daher differenzierter darzustellen. Die Addition der Rohwerte erscheint theoretisch legitim, da beide Tests in ihrer Konzeption und Durchführung nahezu identisch sind. Beide enthalten zur Benennung fotografische Darstellungen und unterscheiden sich v. a. in ihrem Schwierigkeitsgrad aufgrund ihrer Konzeption für das unterschiedliche Alter. Empirisch korrelieren die Rohwerte beider Tests mit $r=$ .84 ( $p$.001). Jedoch könnte aufgrund möglicher empirischer Verteilungsunterschiede zwischen beiden Tests die Addition der Rohwerte zu verzerrten Ergebnissen führen. Daher addierten wir zusätzlich die z-standardisierten Rohwerte zu einer Variable sowie berechneten anhand einer Hauptkomponentenanalyse die Werte der ersten unrotierten Komponente, die durch Verteilungsunterschiede weniger verzerrt sind. Beide neu entstandenen Variablen korrelierten jedoch mit dem Summenscore nahezu perfekt $(r=.988$ bzw. $r=.999)$, weshalb die drei Variablen im statistischen Sinn austauschbar sind. Somit gehen wir von keiner Verzerrung aufgrund von Verteilungsunterschieden aus. Da die ursprüngliche Variable im Gegensatz zu den beiden standardisierten Variablen den Vorteil hat, dass die unstandardisierten Regressionsgewichte intuitiv interpretierbar sind, berichten wir ausschließlich die Ergebnisse mit dem Summenscore als abhängige Variable.

Fehlende Werte traten in der Variable Kontaktdauer (6.65\%) auf, die in 20 Datensätzen multipel imputiert wurden. Die Ergebnisse wurden entsprechend der Empfehlungen von Baraldi und Enders (2010) gepoolt. Für alle statistischen Analysen verwendeten wir SPSS 26, bis auf die Prüfung der Unterschiede zwischen den standardisierten Regressionskoeffizienten, für die wir die Funktion linearHypothesis für multipel imputierte Datensätze aus dem R-Paket carEx (Fox, Weisberg, Price \& Monette, 2019) nutzten.

Zur Untersuchung des Einflusses der Erwerbsbedingungen auf den expressiven Wortschatz wurde zunächst 
geprüft, ob nicht-lineare Zusammenhangsmodelle höhere Vorhersagewerte zeigen als lineare Zusammenhangsmodelle. Wir verglichen die Vorhersagewerte für lineare, logarithmische, zusammengesetzte, s-förmige, exponentielle und logistische Modelle. Der Zusammenhang für den expressiven Wortschatz und das Alter war durch das lineare Modell nicht schlechter vorhersagbar (korr. $R^{2}=$ .33) als durch nicht-lineare Zusammenhänge (höchster Vorhersagewert durch das logarithmische Modell mit einem korr. $\left.R^{2}=.34\right)$. Gleiches galt für den Zusammenhang zwischen dem expressiven Wortschatz und der Kontaktdauer. Der Vorhersagewert für das lineare Modell (korr. $\left.R^{2}=.29\right)$ war nicht schwächer als die für die nicht-linearen Modelle (höchster Vorhersagewert durch das logarithmische Modell mit einem korr. $R^{2}=.29$ ). Daher nehmen wir im Sinne des Prinzips der Parsimonie lediglich einen linearen Zusammenhang an.

Die Ermittlung des Einflusses der Erwerbsbedingungen erfolgte durch eine schrittweise lineare Regressionsanalyse mit den expressiven Wortschatzleistungen als abhängiger Variable und dem am Mittelwert zentrierten $\mathrm{Al}-$ ter in Jahren $(M=8.01$ Jahre $)$ als Prädiktor in Schritt 1 sowie den am Mittelwert zentrierten Kontaktjahren zu Deutsch $(M=6.14$ Jahre $)$ als Prädiktor in Schritt 2. Zur Überprüfung, inwiefern der Einfluss des Alters unter dem Einfluss der Kontaktdauer variiert, fügten wir in Schritt 3 den Interaktionsterm aus dem zentrierten Alter und den zentrierten Kontaktjahren zu Deutsch hinzu. Die Zentrierung der Prädiktoren hat den Vorteil, dass ein möglicher Interaktionseffekt besser interpretierbar ist, $d$. h. sowohl die Steigung als auch der Achsenabschnitt für bestimmte Werte aus der Regressionsgleichung abzulesen sind.

\section{Ergebnisse}

Die mehrsprachigen Grundschulkinder zeigen im expressiven Wortschatz einen mittleren Summenscore von 75.20 ( $S D=31.27$, Min. 3, Max. 144; Median = 79). Die Verteilung des Summenscores zeigt weder Decken- noch Bodeneffekte. Die Korrelation zwischen den Prädiktoren Kontaktjahre und Alter $(r(449)=.58, p<.001)$ entspricht einem starken Effekt. Die Korrelationen zwischen dem expressiven Wortschatz und dem Alter $(r(449)=.57$, $p<.001)$ sowie dem expressiven Wortschatz und den Kontaktjahren $(r(449)=.54, p<.001)$ sind ebenfalls stark und unterscheiden sich nicht signifikant voneinander $(z=0.88, p=.191)$.

\section{Einfluss von Alter und Kontaktdauer}

Zunächst prüften wir, ob das Alter sowie die Kontaktdauer einen Effekt auf die expressiven Wortschatzleistungen haben und wie stark die Effekte im Vergleich sind. Des Weiteren betrachteten wir, ob die Hinzunahme der Kontaktdauer als zusätzliche Erwerbsbedingung zum Alter die Vorhersage der expressiven Wortschatzleistungen erhöht. Die schrittweise lineare Regression ist in Tabelle 1 dargestellt. In Schritt 1 ist das Alter ein bedeutsamer Einflussfaktor auf die expressiven Wortschatzleistungen. Das Alter klärt $32.7 \%$ der Varianz auf, was einem starken Effekt entspricht $(F(1,449)=219.82, p<.001)$. Demnach betrug der Mittelwert des Summenscores für Kinder im Alter von 8.01 Jahren 75.15 Rohwertpunkte. Ein Jahr ältere Kinder weisen im Mittel einen um 14.26 Rohwertpunkte höheren Summenscore auf.

In Schritt 2 sind das Alter und die Kontaktjahre zu Deutsch bedeutsame Einflussfaktoren auf die expressiven Wortschatzleistungen. Sie klären gemeinsam $39.0 \%$ der Varianz auf, was einem starken Effekt entspricht $(F(2,448)=144.72, p<.001)$. Die Vorhersage der expressiven Wortschatzleistungen durch das Alter steigert sich durch die Aufnahme der Kontaktjahre um 6.4\% $(F(1,448)=47.07, p<.001)$. Werden die Schritte vertauscht (in der Tabelle nicht dargestellt), also das Alter als zweiter Prädiktor nach den Kontaktjahren aufgenommen, steigt die Varianzaufklärung um $10.5 \%(F(1,448)=77.07$, $p<.001)$. Demnach sind beide Erwerbsbedingungen inkrementell valide. Der inferenzstatistische Vergleich der beiden Beta-Koeffizienten zeigt, dass sich die Einflüsse von Alter $(\beta=.40, p<.001)$ und Kontaktjahre $(\beta=.31$, $p<$.001) auf die expressiven Wortschatzleistungen nicht signifikant unterscheiden $(F(1,446)=0.84, p=.361)$.

\section{Moderatoreffekt der Kontaktdauer}

Des Weiteren analysierten wir, ob der Effekt des Alters auf die expressiven Wortschatzleistungen unter dem Einfluss der Kontaktdauer variiert. Das Ergebnis der schrittweisen linearen Regressionsanalyse in Schritt 3 aus Tabelle 1 zeigt einen signifikanten Interaktionsterm, der einen eigenen Varianzanteil von $1.6 \%$ der Gesamtvarianz aufklärt $(F(1,447)=11.84, p=.001)$. Die drei Prädiktoren klären zusammen $40.4 \%$ der Varianz auf, was einem starken Effekt entspricht $(F(3,447)=102.76, p<.001)$. Somit moderiert die Kontaktdauer den Einfluss des Alters auf die expressiven Wortschatzleistungen. Kinder mit längerer Kontaktdauer unterscheiden sich weniger von einem Jahr älteren und gleichzeitig einem Kontaktjahr weiter fortgeschrittenen Kindern als Kinder mit kürzerer Kontaktdauer. 
Der Moderatoreffekt ist in Abbildung 1 für ein konkretes Beispiel illustriert. Die bedingten Achsenabschnitte und die bedingte Steigung der Regressionsgeraden für die exemplarischen Werte von -2, 0 und 2 zentrierten Kontaktjahren (was den Werten von 4.14, 6.14 und 8.14 Kontaktjahren entspricht) lassen sich mit Hilfe der Regressionsparameter anhand der folgenden Formel berechnen:

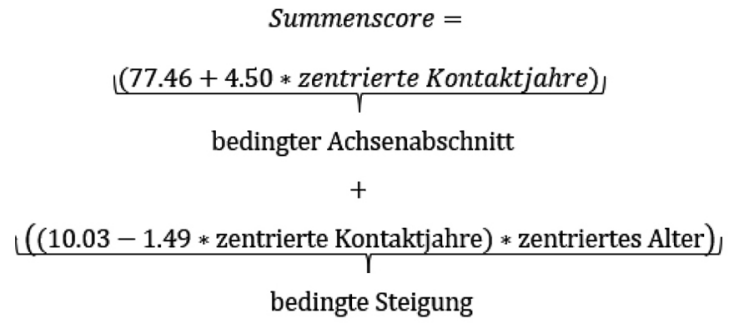

Daraus ergibt sich für die bedingte Steigung, dass Kinder mit 6.14 Kontaktjahren (zentrierte Kontaktjahre 0) verglichen mit einem Jahr älteren und gleichzeitig zwölf Kontaktmonaten weiter fortgeschrittenen Kindern (zentriertes Alter 1) im Mittel 10.03 Rohwertpunkte weniger aufweisen. Kinder mit 8.14 Kontaktjahren (zentrierte Kontaktjahre 2) unterliegen den einem Jahr älteren und gleichzeitig zwölf Kontaktmonaten weiter fortgeschrittenen Kindern im Mittel um 7.05 Rohwertpunkte, und Kinder mit 4.14 Kontaktjahren (zentrierte Kontaktjahre -2) verfügen im Mittel um 13.01 Rohwertpunkte weniger. Demnach ist der Unterschied in den expressiven Wortschatzleistungen von Kindern mit kürzerer Kontaktdauer zu einem Jahr älteren und gleichzeitig einem Kontaktjahr weiter fortgeschrittenen Kindern größer als der entsprechende Unterschied bei Kindern mit längerer Kontaktdauer.

\section{Diskussion}

Um zu klären, ob das Alter und die Kontaktdauer sich als potentielle Bestandteile mehrspracherwerbssensibler Normen für die expressiven Wortschatzleistungen in der Umgebungssprache mehrsprachiger Grundschulkinder nachweisen lassen, untersuchten wir, wie der Einfluss der Kontaktdauer sowie des Alters auf die expressiven Wortschatzleistungen beschaffen ist. Sowohl das Alter als auch die Kontaktdauer klärten jeweils einen eigenen Anteil an der Varianz der expressiven Wortschatzleistungen auf. Die Berücksichtigung beider Prädiktoren konnte die Vorhersage der expressiven Wortschatzleistungen im Vergleich zur isolierten Berücksichtigung nur eines Prädiktors noch einmal bedeutsam steigern.

Der signifikante Einfluss des Alters entspricht unserer Hypothese und steht im Einklang mit bisherigen Befunden 


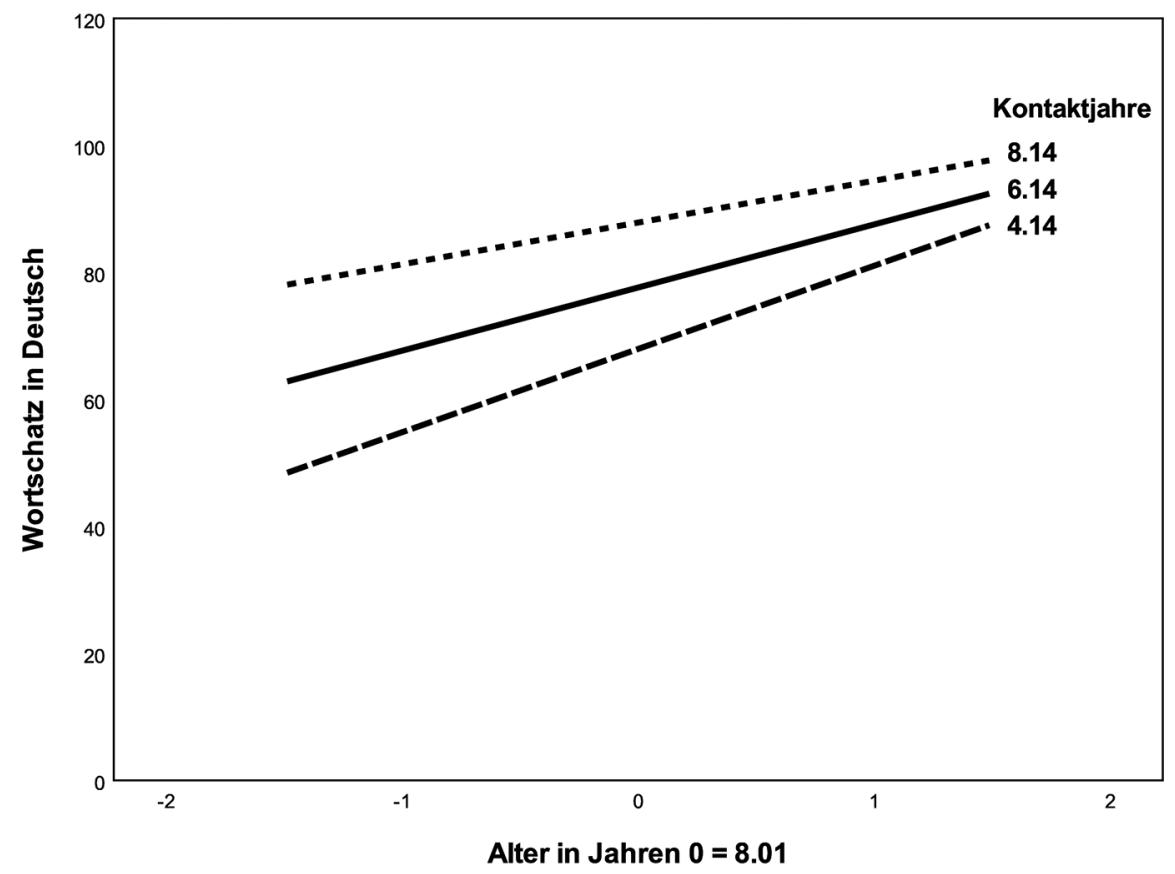

Abbildung 1. Differentieller Zusammenhang von expressiven Wortschatzleistungen in Deutsch und Alter für drei exemplarische Kontaktjahreswerte. zum rezeptiven Wortschatz (Gathercole \& Thomas, 2009; Paradis, 2011; Thordardottir, 2019). Ältere und damit in der Regel kognitiv reifere Kinder haben einen größeren expressiven Wortschatz. Wir konnten in unserer Studie für mehrsprachige Kinder im Grundschulalter zeigen, dass das Alter einen bedeutsamen Einflussfaktor auf die expressiven Wortschatzleistungen darstellt. Jedoch könnte die alleinige Berücksichtigung des Alters in Normgruppen mehrsprachiger Kinder zu kurz greifen, da sie zu einer sehr großen Varianz in der Kontaktdauer führt. Beispielsweise konnten in unserer Stichprobe Kinder im Alter von 7 Jahren zwischen ein bis fünf Kontaktjahre aufweisen. Eine allein altersnormierte Auswertung könnte daher bei mehrsprachigen Kindern durch die Heterogenität bezüglich der Kontaktdauer zu einer verzerrten Interpretation führen.

Der signifikante Einfluss der Kontaktdauer entspricht ebenfalls unserer Hypothese und steht im Einklang mit anderen Studien, die bereits den bedeutsamen Einfluss der Kontaktmonate zur Umgebungssprache auf den rezeptiven (Paradis, 2011) sowie auf den expressiven Wortschatz (Geist, 2017) 5- bis 6-Jähriger Kinder zeigen konnten. Wir wiesen in unserer Studie nach, dass auch für mehrsprachige Kinder im Grundschulalter eine längere Kontaktdauer mit höheren expressiven Wortschatzleistungen einhergeht. Jedoch könnte auch die alleinige Berücksichtigung der Kontaktdauer in Normgruppen mehrsprachiger Kinder zu kurz greifen, da sie zu einer großen Varianz im Alter führt. Beispielsweise schwankte in unserer Stichprobe das Alter der Kinder mit vier Kontaktjahren zwischen fünf und zehn Jahren. Eine allein kontaktdauernormierte Auswertung könnte daher bei mehrsprachigen Kindern durch die Heterogenität bezüglich des Alters ebenfalls zu einer Fehlinterpretation führen.

Der Befund, dass die Berücksichtigung der Kontaktdauer zusätzlich zum Alter die Vorhersage des expressiven Wortschatzes im Vergleich zur singulären Vorhersage durch das Alter oder die Kontaktdauer bedeutsam steigern kann, deutet darauf hin, dass es für die Entwicklung mehrspracherwerbssensibler Normen für expressive Wortschatzleistungen empfehlenswert sein könnte, sowohl das Alter und damit die kognitive Reife, als auch die Kontaktdauer als Anhaltspunkt für die Inputmenge zu berücksichtigen. Dadurch wären Kinder einer Normgruppe hinsichtlich der zentralen Einflussfaktoren Alter und Kontaktdauer deutlich homogener. Dies könnte die Validität der sprachdiagnostischen Entscheidungen erhöhen.

Die Einflüsse der Kontaktdauer sowie des Alters auf die expressiven Wortschatzleistungen waren in unserer Studie vergleichbar groß. Dieses Ergebnis unterscheidet sich von Paradis (2011). Hier hatte die Kontaktdauer deskriptiv einen geringfügig größeren Einfluss als das Alter, auch wenn der Unterschied nicht inferenzstatistisch geprüft wurde. Es wäre interessant zu untersuchen, ob der Unterschied signifikant ist und ggf. mit der Modalität des Wortschatzes (expressiv versus rezeptiv) erklärt werden kann.

Der Befund, dass der Einfluss des Alters durch die Kontaktdauer moderiert wird, entspricht ebenfalls unserer Hypothese sowie den Ergebnissen, die für die expressiven und rezeptiven Wortschatzleistungen von Cobo-Lewis et al. (2010) gefunden wurden. Dieser Befund deutet darauf hin, 
dass der expressive Wortschatzerwerb in der Umgebungssprache in einem früheren Stadium schneller verläuft und sich später verlangsamt. Da der Effekt jedoch zusätzlich lediglich $1.6 \%$ der Varianz aufklärt, wäre ein erneuter Nachweis in Längsschnittstudien ratsam. Insgesamt liefert auch dieser Befund ein starkes empirisches Argument für die gleichzeitige Berücksichtigung des Alters und der Kontaktdauer in mehrspracherwerbssensiblen Normen.

\section{Möglichkeiten und Grenzen mehrspracherwerbssensibler Normen}

Mehrspracherwerbssensible Normen haben das Ziel, die diagnostisch sinnvollen und in Normen realisierbaren Erwerbsbedingungen Alter und Kontaktdauer zu berücksichtigen. Allerdings sind mehrsprachige Kinder eine besonders heterogene Gruppe. Sie unterscheiden sich in vielen Faktoren, die sich auf die sprachlichen Leistungen auswirken und mit ihnen in Wechselwirkung stehen können. Dazu gehören u. a. eine unterschiedliche familiäre Sprachverwendung, verschiedene Nichtumgebungssprachen sowie eine unterschiedliche Nähe der Nichtumgebungssprachen zur Umgebungssprache, verschieden große Inputmengen in der Umgebungs- und Nichtumgebungssprache sowie Unterschiede in der angebotenen Inputqualität (Unsworth, 2016b). Der Einfluss der familiären Sprachverwendung auf die Sprach- und Schriftsprachleistungen in der Umgebungssprache wurde $u$. a. an Analysen der Normstichproben mehrerer sprachdiagnostischer Verfahren belegt. Kinder, die zu Hause kein Deutsch sprachen, unterschieden sich besonders stark von einsprachigen Kindern, aber auch von Kindern, die zu Hause Deutsch und eine andere Sprache sprachen ( Lenhard, Lenhard, Segerer \& Suggate, 2015; Lenhard \& Lenhard, 2017). Unsere Analysen nehmen keine direkte Unterscheidung hinsichtlich der familiären Sprachverwendung der Umgebungssprache vor. Allerdings nehmen wir an, dass die Kontaktdauer zur Umgebungssprache indirekt die familiäre Sprachverwendung berücksichtigt, da in der Regel die Schulkinder einer bestimmten Altersstufe mit verhältnismäßig kurzer Kontaktdauer die Kinder sind, die überwiegend über institutionelle Einrichtungen mit der Umgebungssprache in Kontakt kommen, während die Kinder derselben Altersstufe mit verhältnismäßig langer Kontaktdauer die Kinder sind, die schon seit der frühen Kindheit auch zu Hause regelmäßig mit der Umgebungssprache in Kontakt kommen (De Houwer, 2018; Paradis, 2010). Demnach ist ein großer Zusammenhang zwischen der Sprachverwendung der Umgebungssprache im Elternhaus und der Kontaktdauer zur Umgebungssprache anzunehmen, was die gleichzeitige Berücksichtigung der beiden Variablen als Prädiktoren in Regressionsanalyen wegen Problemen der Multikollinearität erschwert. Von beiden
Variablen erscheint uns die Kontaktdauer für die Realisierung in Normen aufgrund ihrer intervallskalierten Abstufung im Vergleich zur dichotomen Unterscheidung der Sprachverwendung differenzierter. Die Frage, ob die Verwendung der Umgebungssprache im Elternhaus oder die Kontaktdauer zur Umgebungssprache der bessere Prädiktor für die Wortschatzleistungen in der Umgebungssprache ist, sollte jedoch in Folgestudien geklärt werden, da sie für die Entwicklung von Normen höchst relevant ist.

Der Einfluss der unterschiedlichen Nähe der Nichtumgebungssprachen zur Umgebungssprache auf die Sprachleistungen wurde in Cross-linguistischen Studien untersucht (Blom et al., 2020; Kauschke, Lee \& Pae, 2007) und würde für jeweils separate Verfahren für verschiedene Sprachkombinationen sprechen. Die Verfahrensentwicklung wäre dann jedoch vermutlich aufgrund der erforderlichen Stichprobengrößen nur für die häufigen Sprachkombinationen realisierbar, weshalb Kinder mit selteneren Sprachkombinationen benachteiligt wären.

Diese und weitere Einflussfaktoren werden durch die vorgeschlagenen mehrspracherwerbssensiblen Normen nicht oder nur indirekt berücksichtigt. Daher ist es bei der Sprachdiagnostik mehrsprachiger Kinder unverzichtbar, zusätzliche Elternangaben zur Sprachentwicklung, zum Sprachgebrauch und möglichst detailliert zu den Inputbedingungen (Kontaktmonate, Inputmenge usw.) zu erfragen (De Houwer, 2018; Ritterfeld \& Lüke, 2013) und diese Angaben bei der Interpretation mitzuberücksichtigen. Z. B. könnte ein auffälliges Testergebnis bei einem Kind, das neben dem Schulkontext auch im Elternhaus und bei Freizeitaktivitäten weiteren Input in der Umgebungssprache erhält, eindeutiger zu interpretieren sein als bei einem Kind, das bis auf den Schulkontext kaum weiteren Input in der Umgebungssprache erhält.

Bei der Verwendung der Kontaktdauer in der Sprachdiagnostik müssen zudem mindestens drei weitere Aspekte beachtet werden. Erstens ist der Erwerbsbeginn, der für die Bestimmung der Kontaktdauer ermittelt werden muss, nur schwer zu definieren. So wären als Erwerbsbeginn verschiedene Erwerbssituationen und verschiedene Inputvariationen denkbar wie beispielsweise der Eintritt in eine Kindertagesstätte, persönlich an das Kind gerichtete Sprache im Elternhaus, Fernsehkonsum, Hörspiele und Lieder (De Houwer, 2018). Dabei ist diskutabel und für Eltern häufig schwer definierbar, welcher Input als Erwerbsbeginn gezählt werden kann. Zweitens stellt die Kontaktdauer einen recht groben Indikator für die Inputmenge dar (De Houwer, 2018). So können zwei mehrsprachige Kinder mit der gleichen Anzahl an Kontaktmonaten zur Umgebungssprache eine sehr unterschiedliche Inputmenge in der Umgebungssprache erhalten, wenn beispielsweise ein Kind eine Kindertagesstätte vier Stunden pro Tag und ein anderes Kind diese acht Stunden pro Tag besucht. Die Erfas- 
sung der Kontaktdauer über die Anzahl der Stunden, die ein Kind Kontakt zu einer Sprache hatte, wurde daher in der Mehrsprachigkeitsforschung eingeführt (Thordardottir, Rothenberg, Rivard \& Naves, 2006; Unsworth, 2013), was jedoch zum dritten Problem bei der Anwendung der Kontaktdauer in der Sprachdiagnostik führt. Drittens könnte sich ein Validitätsproblem bei der retrospektiven Einschätzung der Kontaktdauer ergeben. Es könnte Eltern schwerfallen, die Kontaktdauer in Monaten bzw. sogar in Stunden für mehrere zurückliegende Jahre genau einzuschätzen (De Houwer, 2018; Thordardottir et al., 2006). Jedoch konnten zahlreiche Studien zeigen, dass zumindest signifikante $\mathrm{Zu}-$ sammenhänge zwischen den Elternangaben zur Inputmenge und den Sprachkompetenzen ihrer Kinder bestehen (für einen Überblick Paradis, 2017).

Trotz der genannten Schwierigkeiten bei der Verwendung der Kontaktdauer in der Sprachdiagnostik sprechen wir uns dafür aus, diese Erwerbsbedingung in mehrspracherwerbssensible Normen aufzunehmen. Um dem Validitätsproblem bei der Erfassung der Kontaktdauer zu begegnen, wäre es ratsam, den Erwerbsbeginn als systematischen Kontakt, z. B. über betreuende Personen oder Institutionen zu standardisieren (Schulz \& Tracy, 2011). Bei der Frage nach dem Erwerbsbeginn könnten markante Zeitpunkte als Antwortoptionen vorgeschlagen werden wie beispielsweise seit der Geburt, seit Eintritt in die Kindertagesstätte, seit der Einschulung oder zu einem anderen Zeitpunkt. Diese Antwortoptionen könnten auch dem Erinnerungsproblem Abhilfe schaffen, weil diese markanten Zeitpunkte voraussichtlich besser erinnert werden können. Dennoch machen diese Ausführungen zur Verwendung der Kontaktdauer in der Sprachdiagnostik deutlich, dass sie nicht als exakte Zahl, sondern eher als Anhaltspunkt für die Einschätzung der Erwerbsgelegenheiten betrachtet werden muss (Schulz \& Tracy, 2011).

Mehrspracherwerbssensible Normen könnten konkret als separate Normen für die Gruppe der mehrsprachigen Kinder realisiert werden. Ihre Altersnormen könnten nach Kontaktdauerzeiträumen aufgesplittet werden (z. B. Jahresnormen aufgesplittet in Kontaktdauerzeiträume von 12 Monaten). Je geringer die Zeitspannen gewählt würden, desto größere Normierungsstichproben wären erforderlich. Eine weitere Möglichkeit wäre die statistische Berechnung von sogenannten kontinuierlichen Normen, die die Rohwertverteilung über das Alter und potentiell auch über weitere Kovariaten wie beispielsweise die Kontaktdauer hinweg regressionsbasiert modellieren (Lenhard, Lenhard \& Gary, 2019).

\section{Limitationen}

Eine methodische Einschränkung dieser Studie besteht in ihrer Anlage als Querschnittdesign, weshalb Aussagen über die Entwicklung des Wortschatzes in Abhängigkeit von der Kontaktdauer dringend längsschnittlich geprüft werden müssen. Eine weitere Limitation besteht in der Bildung eines Summenscores aus den Rohwerten zweier verschiedener Wortschatztests, auf die aus Ermangelung eines durchgängigen Verfahrens mit einer vergleichbar breiten Schwierigkeitsabstufung zurückgegriffen werden musste. Auch wenn unsere Itemanalyse für den Summenscore eine gute Reliabilität nachweisen konnte und beide Verfahren isoliert Nachweise für ihre Validität an einsprachigen Kindern erbracht haben, fehlt ein Nachweis für die Validität des Summenscores für mehrsprachige Kinder. Zudem ist bisher noch nicht nachgewiesen, ob die Items der für einsprachige Kinder konzipierten expressiven Wortschatztests die Leistungen mehrsprachiger Kinder vergleichbar gut messen können. Hierzu könnten anhand von Messinvarianzanalysen mit ein- versus mehrsprachigen Kindern diejenigen Items eliminiert werden, die sich nicht für die Testung der Umgebungssprache von mehrsprachigen Kindern eignen.

\section{Fazit}

Trotz dieser Einschränkungen konnte diese Studie erstmals einen empirischen Beleg dafür liefern, dass die Erwerbsbedingungen Kontaktdauer und Alter auch als Bestandteile mehrspracherwerbssensibler Normen für expressive Wortschatzleistungen in der Umgebungssprache mehrsprachiger Kinder im Grundschulalter in Erwägung gezogen werden könnten. Daher erscheinen entweder die Ergänzung vorhandener Wortschatztests um eine mehrspracherwerbssensible Normierung oder die Neuentwicklung eines Testverfahrens für den expressiven Wortschatz in der Umgebungssprache Deutsch für mehrsprachige Grundschulkinder inklusive der Entwicklung von mehrspracherwerbssensiblen Normen als sinnvolle Optionen. Ein solches Verfahren wäre in Deutschland einzigartig und würde die Diagnostikmöglichkeiten von Wortschatzleistungen mehrsprachiger Kinder deutlich erweitern. Denn obwohl bei mehrsprachigen Kindern die Sprachdiagnostik in allen Sprachen zu empfehlen ist, kann es zweifelsohne aufgrund von fehlenden Ressourcen vorkommen, dass die Nichtumgebungssprache nicht umfassend untersucht werden kann. Für die Entscheidung, welche mehrsprachigen Kinder in ihren expressiven Wortschatzleistungen in der Umgebungssprache auch im Vergleich zu mehrsprachigen Kindern und somit besonders auffällig sind, könnte ein Verfahren mit den vorgeschlagenen mehrspracherwerbssensiblen 
Normen herangezogen werden. Dieses auffällige Ergebnis im Wortschatz ließe sich dann nutzen, um Unterstützungsmaßnahmen zum Erwerb des Wortschatzes sowie weitere Beobachtungsmaßnahmen $\mathrm{zu}$ begründen und möglicherweise eine tiefergehende Sprachdiagnostik durch Expertinnen und Experten in der Umgebungs- und in der Nichtumgebungssprache sowie in der Sprachverarbeitung zu empfehlen.

\section{Literatur}

AWMF. (2011). Interdisziplinäre S2k-Leitlinie: Diagnostik von Sprachentwicklungsstörungen (SES), unter Berücksichtigung umschriebener Sprachentwicklungsstörungen (USES). Registernummer 049-006. Verfügbar unter: https://www.awmf.org/ leitlinien/detail/ll/049-006.html

Baraldi, A. N. \& Enders, C. K. (2010). An introduction to modern missing data analyses. Journal of School Psychology, 48, 5-37. https://doi.org/10.1016/j.jsp.2009.10.001

Bialystok, E. (2001). Bilingualism in development. Language, literacy, and cognition. Cambridge: Cambridge University Press.

Blom, E., Boerma, T., Bosma, E., Cornips, L., van den Heuij, K. \& Timmermeister, M. (2020). Cross-language distance influences receptive vocabulary outcomes of bilingual children. First Language, 40, 151 -171. https://doi.org/10.1177/0142723719 892794

Cobo-Lewis, A. B., Pearson, B. Z., Eilers, R. E. \& Umbel, V. C. (2010). Effects of bilingualism and bilingual education on oral and written English skills: A multifactor study of standardized test outcomes. In D. K. Oller \& R. E. Eilers (Eds.), Language and literacy in bilingual children (S. 64-97). Clevedon: Multilingual Matters.

Daskalaki, E., Blom, E., Chondrogianni, V. \& Paradis, J. (2020). Effects of parental input quality in child heritage language acquisition. Journal of Child Language, 47, 709-736. https://doi.org/ 10.1017/S0305000919000850

De Houwer, A. (2018). The role of language input environments for language outcomes and language acquisition in young bilingual children. In D. Miller, F. Bayram, J. Rothman \& L. Serratrice (Eds.), Bilingual cognition and language. The state of the science across subfields (S. 127 - 154). Amsterdam: John Benjamins.

Ehl, B., Bruns, G. \& Grosche, M. (2020). Differentiated bilingual vocabulary assessment reveals similarities and differences compared to monolinguals: Conceptual versus single-language scoring and the relation with home language and literacy activities. International Journal of Bilingualism, 24, 715-728. https://doi.org/10.1177/1367006919876994

Ertanir, B., Kratzmann, J. \& Sachse, S. (2019). Sozio-emotionale Kompetenzen mehrsprachiger Kindergartenkinder und deren Wechselwirkungen mit den Sprachleistungen im Deutschen. Zeitschrift für Entwicklungspsychologie und Pädagogische Psychologie, 51, 31-44. https://doi.org/10.1026/0049-8637/a00 0207

Fox, J., Weisberg, S., Price, B. \& Monette, G. (2019). carEX: Supplemental and Experimental Functions. $R$ package version 0.2-0/r605. Verfügbar unter: https://R-Forge.R-project.org/ projects/car/

Gathercole, V. C. M. \& Thomas, E. M. (2009). Bilingual first-language development. Dominant language takeover, threatened minority language take-up. Bilingualism: Language and Cogniti- on, 12, 213-237. https://doi.org/10.1017/S136672890 9004015

Gathercole, V. C. M., Thomas, E. M., Roberts, E. J., Hughes, C. O. \& Hughes, E. K. (2013). Why assessment needs to take exposure into account: Vocabulary and grammatical abilities in bilingual children. In V. C. M. Gathercole (Ed.), Issues in the Assessment of Bilinguals (S. 20 -55). Bristol: Channel View Publications.

Geist, B. (2017). Wortschatz von Kindern mit Deutsch als Zweitsprache. Frühe Bildung, 6, 124-132. https://doi.org/10.1026/ 2191-9186/a000326

Glück, C. W. (2011). Wortschatz- und Wortfindungstest für 6- bis 10-Jährige. WWT 6-10. München: Elsevier.

Grosjean, F. (2016). The Complementarity Principle and its impact on processing, acquisition, and dominance. In C. Silva-Corvalán \& J. Treffers-Daller (Eds.), Language dominance in bilinguals. Issues of measurement and operationalization (S. 66-84). Cambridge: Cambridge University Press.

Håkansson, G., Salameh, E.-K. \& Nettelbladt, U. (2003). Measuring language development in bilingual children: Swedish- Arabic children with and without language impairment. Linguistics, 41, 255-288. https://doi.org/10.1515/ling.2003.009

Hamann, C. \& Abed Ibrahim, L. (2017). Methods for identifying specific language impairment in bilingual populations in Germany. Frontiers in Communication, 2, 1-19. https://doi.org/ 10.3389/fcomm.2017.00016

Hammer, C. S., Hoff, E., Uchikoshi, Y., Gillanders, C., Castro, D. \& Sandilos, L. E. (2014). The language and literacy development of young dual language learners. A critical review. Early Childhood Research Quarterly, 29, 715-733. https://doi.org/10.1016/j. ecresq.2014.05.008

Kany, W. \& Schöler, H. (2009). Diagnostik schulischer Lern- und Leistungsschwierigkeiten. Stuttgart: Kohlhammer.

Kauschke, C., Lee, H.-W. \& Pae, S. (2007). Similarities and variation in noun and verb acquisition: A crosslinguistic study of children learning German, Korean, and Turkish. Language and Cognitive Processes, 22, 1045-1072. https://doi.org/10.1080/ 01690960701307348

Kiese-Himmel, C. (2005). Aktiver Wortschatztest für 3- bis 5-jährige Kinder. AWST-R. Göttingen: Beltz-Test.

KMK. (2019). Bildungssprachliche Kompetenzen in der deutschen Sprache stärken. Verfügbar unter: https://www.kmk.org/fileadmin/Dateien/pdf/PresseUndAktuelles/2019/2019-1206_Bildungssprache/2019-368-KMK-Bildungssprache-Beispiele.pdf

Lenhard, A. \& Lenhard, W. (2017). Diagnostik von Lesestörungen mit ELFE /l bei Kindern mit Migrationshintergrund (inklusive adaptierter Normen). Dettelbach: Psychometrica.

Lenhard, A., Lenhard, W. \& Gary, S. (2019). Continuous norming of psychometric tests: A simulation study of parametric and semiparametric approaches. PloS ONE, 14(9), e0222279. https://doi. org/10.1371/journal.pone.0222279

Lenhard, A., Lenhard, W., Segerer, R. \& Suggate, S. (2015). Peabody Picture Vocabulary Test - 4. Ausgabe (deutsche Fassung). Frankfurt am Main: Pearson.

Lowe, H., Henry, L., Müller, L.-M. \& Joffe, V. L. (2018). Vocabulary intervention for adolescents with language disorder: a systematic review. International Journal of Language \& Communication Disorders, 53, 199-217. https://doi.org/10.1111/14606984.12355

Lüke, C., Starke, A. \& Ritterfeld, U. (2020). Sprachentwicklungsdiagnostik bei mehrsprachigen Kindern. In S. Sachse, A.-K. Bockmann \& A. Buschmann (Hrsg.), Sprachentwicklung. Entwicklung - Diagnostik - Förderung im Kleinkind- und Vorschulalter (S. 221 -238). Berlin: Springer. 
Marinis, T., Armon-Lotem, S. \& Pontikas, G. (2017). Language impairment in bilingual children. Linguistic Approaches to Bilingualism, 7, 265 - 276. https://doi.org/10.1075/lab.00001.mar

McElvany, N., El-Khechen, W., Schwabe, F. \& Kessels, U. (2016). Qualitative Wortschatzunterschiede zwischen Mädchen und Jungen im Grundschulalter. Zeitschrift für Pädagogische Psychologie, 30, 45-55. https://doi.org/10.1024/1010-0652/a000 167

Melzer, J., Rißling, J.-K., Lehmkuhl, G. \& Petermann, F. (2018). Sprachentwicklungsdiagnostik. Monatsschrift Kinderheilkunde, 166, 159 -168. https://doi.org/10.1007/s00112-017-0385-y

Neugebauer, U. \& Becker-Mrotzek, M. (2015). Gütemerkmale von 21 Sprachstandsverfahren im Elementarbereich. In G. Esser, M. Hasselhorn \& W. Schneider (Hrsg.), Diagnostik im Vorschulalter (S. 19 - 42). Göttingen u. a.: Hogrefe.

Paradis, J. (2010). The interface between bilingual development and specific language impairment. Applied Psycholinguistics, $31,227-252$.

Paradis, J. (2011). Individual differences in child English second language acquisition. Comparing child-internal and child-external factors. Linguistic Approaches to Bilingualism, 1, 213 237. https://doi.org/10.1075/lab.1.3.01 par

Paradis, J. (2016). The development of English as a second language with and without specific language impairment: Clinical implications. Journal of Speech, Language, and Hearing Research, 59, 171-182. https://doi.org/10.1044/2015_JSLHR-L15-0008

Paradis, J. (2017). Parent report data on input and experience reliably predict bilingual development and this is not trivial. Bilingualism: Language and Cognition, 20, 27 -28. https://doi.org/ 10.1017/S136672891600033X

Paradis, J. (2018). Language-level input factors are not enough to explain child bilingual acquisition. Linguistic Approaches to Bilingualism, 8, 753 - 757. https://doi.org/10.1075/lab.18059.par

Paradis, J., Schneider, P. \& Sorenson Duncan, T. (2013). Discriminating children with language impairment among englishlanguage learners from diverse first-language backgrounds. Journal of Speech, Language, and Hearing Research, 56, $971-$ 981. https://doi.org/10.1044/1092-4388(2012/12-0050)

Peña, E. D., Bedore, L. M. \& Fiestas, C. (2013). Development of bilingual semantic norms: Can two be one? In V. C. M. Gathercole (Hrsg.), Solutions for the assessment of bilinguals (S. $103-$ 124). Bristol: Multilingual Matters.

Reiss, K., Weis, M., Klieme, E. \& Köller, O. (2019). PISA 2018. Münster, New York: Waxmann. https://doi.org/10.31244/ 9783830991007

Reitenbach, V., Schastak, M. \& Rauch, D. (2018). Sprachstandsdiagnostik. In D. B. Maehler, A. Shajek \& H. U. Brinkmann (Hrsg.), Diagnostik bei Migrantinnen und Migranten (S. 95 -149). Göttingen: Hogrefe.

Ritterfeld, U. \& Lüke, C. (2013). Mehrsprachen-Kontexte 2.0. Erfassung der Inputbedingungen von mehrsprachig aufwachsenden Kindern. Verfügbar unter: http://hdl.handle.net/2003/ 31166

Schneider, W., Baumert, J., Becker-Mrotzek, M., Hasselhorn, M., Kammermeyer, G., Rauschenbach, T. et al. (2012). Expertise "Bildung durch Sprache und Schrift (BISS)". Verfügbar unter: https://www.bmbf.de/files/BISS_Expertise.pdf

Schulz, P. \& Tracy, R. (2011). LiSe-DaZ. Linguistische Sprachstandserhebung - Deutsch als Zweitsprache. Göttingen u.a.: Hogrefe.

Seifert, S., Paleczek, L., Schwab, S. \& Gasteiger-Klicpera, B. (2017). GraWo. Grazer Wortschatztest. Göttingen: Hogrefe.

Settinieri, J. \& Jeuk, S. (2019). Einführung in die Sprachdiagnostik. In S. Jeuk \& J. Settinieri (Hrsg.), Sprachdiagnostik Deutsch als Zweitsprache (S. 3 -20). Berlin: De Gruyter.
Teoh, W. Q., Brebner, C. \& McAllister, S. (2018). Bilingual assessment practices: challenges faced by speech-language pathologists working with a predominantly bilingual population. Speech, Language and Hearing, 21, 10-21. https://doi.org/ 10.1080/2050571X.2017.1309788

Thordardottir, E. (2011). The relationship between bilingual exposure and vocabulary development. International Journal of Bilingualism, $\quad 15, \quad 426-445 . \quad$ https://doi.org/10.1177/ 1367006911403202

Thordardottir, E. (2019). Amount trumps timing in bilingual vocabulary acquisition. Effects of input in simultaneous and sequential school-age bilinguals. International Journal of Bilingualism, 23, 236-255. https://doi.org/10.1177/1367006917 722418

Thordardottir, E., Rothenberg, A., Rivard, M.-E. \& Naves, R. (2006). Bilingual assessment: Can overall proficiency be estimated from separate measurement of two languages? Journal of Multilingual Communication Disorders, 4, 1-21. https://doi.org/ 10.1080/14769670500215647

Torppa, M., Tolvanen, A., Poikkeus, A.-M., Eklund, K., Lerkkanen, M.-K., Leskinen, E. et al. (2007). Reading development subtypes and their early characteristics. Annals of Dyslexia, 57 , 3-32. https://doi.org/10.1007/s11881-007-0003-0

Unsworth, S. (2013). Assessing the role of current and cumulative exposure in simultaneous bilingual acquisition: The case of Dutch gender. Bilingualism: Language and Cognition, 16, $86-$ 110. https://doi.org/10.1017/S1366728912000284

Unsworth, S. (2016a). Early child L2 acquisition: Age or input effects? Neither, or both? Journal of Child Language, 43, 608-634. https://doi.org/10.1017/S030500091500080X

Unsworth, S. (2016b). Quantity and quality of language input in bilingual language development. In E. Nicoladis \& S. Montanari (Eds.), Bilingualism across the lifespan. Factors moderating language proficiency (S. $103-122)$. Berlin: De Gruyter. https:// doi.org/10.1037/14939-007

Vernon-Feagans, L., Bratsch-Hines, M., Reynolds, E. \& Willoughby, M. (2020). How early maternal language input varies by race and education and predicts later child language. Child Development, 91, 1098-1115. https://doi.org/10.1111/ cdev.13281

Weiß, R. H. \& Osterland, J. (2012). CFT 1-R. Grundintelligenztest Skala 1 - Revision. Göttingen u. a.: Hogrefe.

Wong Fillmore, L. (2000). Second-language learning in children: A model of language learning in social context. In E. Bialystok (Ed.), Language processing in bilingual children (S. 49-69). Cambridge: Cambridge University Press.

\section{Förderung}

Das diesem Beitrag zugrundeliegende Vorhaben wurde u.a. mit Mitteln des Bundesministums für Bildung und Forschung (BMBF) unter dem Förderkennzeichen 01JI1501C gefördert. Die Verantwortung für den Inhalt dieser Veröffentlichung liegt bei der Autorengruppe.

Open Access-Veröffentlichung ermöglicht durch die Bergische Universität Wuppertal. 
ORCID

Birgit Ehl

iDhttps://orcid.org/0000-0002-1173-2827

Michael Grosche

(iD) https://orcid.org/0000-0001-6646-9184

\section{Birgit Ehl}

Bergische Universität Wuppertal

Institut für Bildungsforschung

in der School of Education

Gaußstraße 20

42119 Wuppertal

Germany

birgit.ehl@uni-wuppertal.de 